\title{
Oncological Outcomes of Patients with Peritoneal Metastasis-only Colorectal Cancer Treated with First-line Bevacizumab and FOLFIRI through Irinotecan Dose Escalation According to UGT1A1 Polymorphism: Compared to Liver Metastasis-only, and Lung Metastasis-only
}

Ching-Chun Li

Kaohsiung Medical University https://orcid.org/0000-0001-8459-2426

Tsung-Kun Chang

Kaohsiung Medical University Hospital: Kaohsiung Medical University Chung Ho Memorial Hospital

Yen-Cheng Chen

Kaohsiung Medical University Hospital: Kaohsiung Medical University Chung Ho Memorial Hospital

Hsiang-Lin Tsai

Kaohsiung Medical University Hospital: Kaohsiung Medical University Chung Ho Memorial Hospital

Ching-Wen Huang

Kaohsiung Medical University Hospital: Kaohsiung Medical University Chung Ho Memorial Hospital

Wei-Chih Su

Kaohsiung Medical University Hospital: Kaohsiung Medical University Chung Ho Memorial Hospital Cheng-Jen Ma

Kaohsiung Medical University Hospital: Kaohsiung Medical University Chung Ho Memorial Hospital

Tzu-Chieh Yin

Kaohsiung Municipal Ta-Tung Hospital: Kaohsiung Municipal United Hospital

Po-Jung Chen

Kaohsiung Municipal Hsiao-kang Hospital: Kaohsiung Municipal Siaogang Hospital Jaw-Yuan Wang ( $\sim$ cy614112@ms14.hinet.net)

Kaohsiung Medical University Hospital https://orcid.org/0000-0002-7705-2621

Research article

Keywords: Prognosis, Metastatic colorectal cancer, Peritoneal carcinomatosis-only, UGT1A1, Irinotecan dose escalation

Posted Date: July 14th, 2021 
DOl: https://doi.org/10.21203/rs.3.rs-708537/v1

License: (c) (1) This work is licensed under a Creative Commons Attribution 4.0 International License. Read Full License 


\section{Abstract}

\section{Background:}

The prognosis of metastatic colorectal cancer (mCRC) depends on the metastatic site and systemic therapy regimen. Peritoneal metastases are associated with a relatively unfavorable prognosis among patients with $\mathrm{mCRC}$. In this article, we present the treatment outcomes of patients with peritoneal carcinomatosis (PC) - only, liver metastasis (LiM)-only, and lung metastasis (LuM)-only CRC.

\section{Methods:}

Overall, $206 \mathrm{mCRC}$ patients with single-site metastasis and who had received treatment from January 2014 to December 2018 were recruited. Among 206 patients with mCRC, 15 had PC-only mCRC, 145 had LiM-only mCRC, and 46 had LuM-only mCRC. They attended regular follow-ups until November 2020, and the median follow-up period was 24.7 months (5.1-41.3 months). Patients' characteristics, including clinical data, gene mutation profiles, and clinical outcomes, were evaluated. All patients with PC-only CRC were treated with first-line bevacizumab and FOLFIRI, and the irinotecan dose escalation depended on UGT1A1 polymorphism.

\section{Results:}

Of the 206 patients, no statistical difference was observed between the PC-only, LiM-only, and LuM-only groups in terms of age, primary tumor location, RAS mutation status, $B R A F$ mutation status, and epidermal growth factor receptor overexpression (all $P>0.05$ ). KRAS mutations were detected in two (16.7\%) patients with PC-only CRC, 39 (36.4\%) patients with LiM-only CRC, and 12 (36.4\%) patients with LuM-only CRC. NRAS mutations were detected in one (8.3\%) patient with PC-only CRC, six (7.3\%) patients with LiM-only CRC, and two (7.1\%) patients with LuM-only CRC. BRAF mutations were detected in two (15.4\%) patients with PC-only CRC, seven (6.9\%) patients with LiM-only CRC, and one (2.9\%) patient with LuM-only CRC. Patients with PC-only CRC had a median progression-free survival (mPFS) of 18.0 months and a median overall survival (mOS) of 24.6 months. Patients with LiM-only or LuM-only CRC had mPFS of 18.2 and 26.6 months and mOS of 25.0 and 44.5 months, respectively. No significant differences regarding PFS and OS (both $P>0.05$ ) between the three groups of patients with mCRC were observed.

\section{Conclusions:}

A PC-only status is considered to be a challenge in the treatment of patients with mCRC. Our study revealed that in patients with PC-only $\mathrm{mCRC}$ had a higher incidence of $B R A F$ mutations, and treatment of first-line bevacizumab and FOLFIRI through irinotecan dose escalation according to UGT1A1 polymorphism could confer such patients with comparable outcomes to that of patients with LiM-only and LuM-only mCRC. However, further prospective randomized trials on patients with peritoneal metastatic mCRC should be conducted to verify the findings of this retrospective study. 


\section{Introduction}

Colorectal cancer (CRC) is a major cause of cancer-related deaths worldwide; over 1 million new cases are diagnosed annually [1-2]. Approximately $20 \%-25 \%$ of patients with CRC present with metastases at their initial diagnosis, and the most common sites of metastases from CRC are the liver, lung, and peritoneum [3]. The prognosis of metastatic colorectal cancer ( $\mathrm{mCRC}$ ) depends on the specific metastatic sites involved; a combination of systemic therapy and metastasectomy could provide the best survival rate $[4,5]$. Peritoneal carcinomatosis $(P C)$ - only $C R C$ has a poor prognosis and is often considered to be a terminal condition. A comparison between isolated site metastasis between peritoneum, liver, and lung metastases reported median overall survival (OS) periods of 16.3 months in PC-only CRC, 19.1 months in liver metastasis CRC, and 24.6 months in lung metastasis CRC [6].

Treatment of $\mathrm{mCRC}$ has significantly improved in terms of progression-free survival (PFS) and OS due to the use of new chemotherapeutic and molecular target agents. The association between clinical outcomes and the gene mutations of the Kras, NRAS, and BRAF genes has become more clearly understood. RAS mutation status has been reported to exhibit a significant association with the survival outcomes of patients with liver metastasis (LiM)-only mCRC [7].

The current standard first-line chemotherapy for patients with mCRC includes fluoropyrimidine-based doublet chemotherapy-FOLFOX or FOLFIRI-plus bevacizumab [3]. A retrospective study revealed that patients with $\mathrm{mCRC}$ who received escalated doses of irinotecan according to uridine diphosphate glucuronosyltransferase 1A1 (UGT1A1) genotyping exhibited favorable clinical responses and outcomes [8]. Recently, our prospective, randomized study also suggested that if patients with $\mathrm{mCRC}$, regardless of their KRAS gene status, receive UGT1A1 genotyping, they can tolerate escalated doses of irinotecan and potentially achieve a more favorable clinical outcome without significantly increased toxicity [9, 12]. Furthermore, the oncological outcomes of patients with BRAF-mutated mCRC treated using FOLFIRI plus bevacizumab with irinotecan dose escalation as a first-line therapy are acceptable with tolerable adverse events; this approach may be a feasible treatment option for such patients [10]. In the current retrospective study, we investigated the clinical outcomes of patients with PC-only $\mathrm{MCRC}$ who received first-line bevacizumab and FOLFIRI with irinotecan dose escalation according to UGT1A1 polymorphism.

\section{Methods}

\section{Patients' characteristics}

From a single institution, 398 patients with stage IV CRC who had received treatment between January 2014 and December 2018 were selected. Patients with record duplications $(n=5)$ or neuroendocrine tumors $(n=1)$ were excluded, and those with two metastatic sites $(n=159)$ or other metastatic sites $(n=$ 16) were also excluded. For the study, 15 (15/398, 3.8\%) patients consecutively diagnosed as having PConly CRC, 145 (145/398, 36.4\%) patients diagnosed as having LiM-only CRC, and 46 (46/398, 11.6\%) patients diagnosed were as having lung metastasis (LuM)-only CRC were recruited; the flow diagram of 
patient selection is presented in Figure 1. A case series analysis was performed using a routinely updated and maintained electronic medical record database. Demographic data included age at diagnosis, sex, primary tumor location, and gene mutation. All aspects of this study were approved by the institutional review board of Kaohsiung Medical University Hospital. Finally, this study was a retrospective review of 206 patients with mCRC, and written informed consent was obtained from all patients (KMUHIRB$\mathrm{E}(\mathrm{I})$-20200036). The responses were classified by a radiologist according to RECIST Version 1.1 [10]. DNA extraction for direct sequencing of KRAS/NRAS and immunohistochemical (IHC) analysis of epidermal growth factor receptor (EGFR) expression were performed using the methods described in our previous study [9].

\section{Oncological follow-up}

These patients were followed up for a median of 24.7 months (range, 5.1-41.3 months). Follow-ups included physical examinations and carcinoembryonic antigen measurements every 3 months for the first 2 years and twice a year thereafter. A computed tomography (CT) scan of the abdomen and thorax was arranged every 3 months for the first 3 years and every 3-6 months thereafter. We performed colonoscopies within 1 year after surgery and held follow-ups at 3-year intervals. Magnetic resonance imaging and positron emission CT were only used when necessary.

\section{Statistical analysis}

Data were analyzed using SPSS version 20.0 (SPSS, Inc., Chicago, IL, USA). PFS and OS rates were plotted using the Kaplan-Meier method. PFS was defined as the time from the start date of treatment until the date of any type of progression or the final follow-up, and OS was defined as the time from the beginning of treatment to death from any cause or to the final follow-up. PFS and OS were evaluated using the Kaplan-Meier method, and the log-rank test was used to compare time-to-event distributions. Statistical significance was set at $P<0.05$.

\section{Results}

\section{Patient series, tumor characteristics, and mutation status}

In total, 206 patients with single-site mCRC were included in the analysis. Of these, $15(15 / 206,7.3 \%)$ patients were subsequently diagnosed as having PC-only CRC, 145 (145/206, 70.4\%) were were diagnosed as having LiM-only CRC, and 46 (46/206, 22.3\%) were diagnosed as having LuM-only CRC. The median age was 65.0 years (range, 37-71 years) in the PC-only group, 62.0 years (range, 26-90 years) in the LiM-only group, and 65.5 years (range, $41-86$ years) in the LuM-only group. The status of LiM-only or LuM-only patients was not related to primary tumor location $(P=0.191)$. Moreover, in 109 (75.1\%) patients with LiM-only CRC and 33 (71.7\%) patients with LuM-only CRC, the primary tumors originated from the left colon. A comparable rate of occurrence from the right or left colon in patients with PC-only CRC was observed; the primary tumors of eight (53.3\%) patients with PC-only CRC originated from the left colon. 
Genotyping of KRAS, NRAS, BRAF, and EGFR as well as overexpression analysis revealed no significant differences in metastatic site (Table 1). KRAS and NRAS mutations were evident in respectively 2 patients (16.7\%) and 1 (8.3\%) patient with PC-only CRC, 39 (36.4\%) and 6 (7.3\%) patients with LiM-only CRC, and $12(36.4 \%)$ and $2(7.1 \%)$ in patients with LuM-only CRC. BRAF and EGFR overexpression was respectively evident in 2 (15.4\%) and 12 (92.3\%) patients with PC-only CRC, 7 (6.9\%) and 76 (89.4\%) patients with LiM-only CRC, and 1 patient (2.9\%) and 16 (84.2\%) patients with LuM-only CRC. FOLFIRI (folinic acid + fluorouracil + irinotecan) plus bevacizumab was the first-line treatment for patients with PC-only CRC, and irinotecan dose escalation was conducted according to UGT1A1 polymorphism [8, 12].

\section{Survival evaluation}

In patients with PC-only CRC, the median PFS was 18.0 months (range, 3.7-38.4 months), and the median OS was 24.6 months (range, 5.1-41.3 months). Among the 15 patients with PC-only CRC, six remained alive at the final follow-up visit conducted in November 2020. During the same period, the PFS of those with LiM-only and LuM-only CRC was 18.2 months and 26.6 months, and the OS of those with LiM-only and LuM-only CRC was 25.0 months and 44.5 months. Patients with PC-only mCRC did not have significantly inferior PFS or OS rates compared with patients with LiM-only or LuM-only mCRC (Figure 2 and 3 , both $P>0.05$ ).

\section{Discussion}

Approximately $20 \%$ of patients with CRC had metastases at their initial diagnosis. Furthermore, in almost $40 \%$ of the remaining patients, their (initially limited) diseases progressed to metastases during treatment [13]. The tumor cell entrapment hypothesis explained how peritoneal metastases occurred through the shedding, binding, migration, and survival of cancer cells [14]. Peritoneal metastasis is associated with higher risks of death from all causes, and the reported median OS after 5-fluorouracil-only systemic chemotherapy is 5 to 7 months [15-16] and varies between 13 and 34 months, depending on the chemotherapeutic and molecular targeting agents used [17-19]. Because the plasma-peritoneal barrier reduces intraperitoneal drug penetration, systemic chemotherapy for PC-only CRC has only limited efficacy. According to a meta-analysis, cytoreductive surgery (CRS) and hyperthermic intraperitoneal chemotherapy (HIPEC) provided survival benefits for select patients with PC from CRC [20]. A summarized analysis of these 76 studies indicated that the median OS was approximately 29 months in the CRS plus HIPEC group. However, the patients were selected according to their favorable performance statusand were able to receive surgical intervention, and the mean mortality and morbidity rates of those in the HIPEC program were 2.8\% and 33.0\%, respectively. In 2021, Quénet et al. reported both the absence of an OS benefit after the addition of HIPEC to CRS and more frequent late postoperative complications; they suggested that CRS alone should be the cornerstone of therapeutic strategies with curative intent for colorectal peritoneal metastases [21]. In our study, the median PFS and OS rates of 15 PC-only CRC patients given first-line treatment of FOLFIRI plus bevacizumab were 18.0 and 24.6 months, respectively, and irinotecan dose escalation depended on UGT1A1 polymorphism. 
Irinotecan must be converted by a carboxylesterase to $\mathrm{SN}-38$, which is actively cytotoxic and is detoxified by glucuronidation activity of uridine diphosphate glucuronosyltransferase (UGT) ; therefore, the UGT1A1 genotype represents the development of drug-associated adverse events (AEs). In a genotype-directed dose-determination study on irinotecan dose escalation in patients given first-line FOLFIRI for $\mathrm{mCRC}$, patients with homozygous UGT1A1*28/*28 developed more severe irinotecan-associated AEs more frequently that did patients with other genotypes [22]. By contrast, the clinical presentations of patients with UGT1A1*1/*28 exhibited individual variations; however, the recommended initial irinotecan dose of $180 \mathrm{mg} / \mathrm{m}^{2}$ was generally well-tolerated in these patients [23]. Conversely, patients with the homozygous UGT1A1*1/*1 genotype had less severe irinotecan-associated $A E s$ and could tolerate an irinotecan dose as high as $260 \mathrm{mg} / \mathrm{m}^{2}$ [24]. Patients with the UGT1A1*1/*1 and *1/*28 genotypes received high doses of irinotecan to achieve a favorable objective response rate (ORR) without major AEs [25]. According to the pan-Asian-adapted European Society for Medical Oncology (ESMO) consensus guidelines, patients with a favorable UGT1A1 genotype (homozygous wild * $1 / * 1$ and heterozygous * $1 / * 28$ ) can tolerate high-dose irinotecan without notable toxicity [26]. In our research, 12 of the 15 patients with PC-only CRC had the UGT1A1*1/*1 genotype; three patients with the UGT1A1*1/*1 genotype received escalated irinotecan doses up to $260 \mathrm{mg} / \mathrm{m}^{2}$, and four patients with the UGT1A1* $1 /{ }^{*} 1$ genotype received escalated irinotecan doses up to 210 or $240 \mathrm{mg} / \mathrm{m}^{2}$ (Table 2 ).

In patients with $\mathrm{mCRC}$, the respective 5-year survival rates of those with resectable hepatic, pulmonary, and peritoneal metastases were approximately $60 \%, 40 \%$, and $20 \%$ [27-31], respectively, and peritoneal metastases were associated with a $20 \%$ reduction in PFS and a $30 \%$ reduction in OS compared with nonperitoneal metastatic $\mathrm{MCRC}$ [32]. Our results demonstrated that clinical outcomes were improved by irinotecan dose escalation according to UGT1A1 polymorphisms in patients with PC-only mCRC treated with first-line bevacizumab and FOLFIRI; the median PFS and OS of patients with PC-only mCRC were 18.0 months and 24.6 months, respectively. Overall, in the present study, patients with PC-only mCRC had comparable PFS and OS to patients with LiM-only or LuM-only CRC. However, ours was only an observational study with a relatively small sample size; future investigations could have a longer followup and larger sample sizes to confirm the findings of our study.

\section{Conclusion}

Although chemotherapy regimens with new chemotherapeutic and molecular targeting agents improved mCRC outcomes, patients with PC-only mCRC were still considered to have poor clinical outcomes. In summary, our research suggest that patients with PC-only $\mathrm{MCRC}$ treated with first-line bevacizumab and FOLFIRI and given irinotecan dose escalation according to UGT1A1 polymorphism might obtain outcomes comparably favorable to those of patients with LiM-only or LuM-only mCRC. Further prospective randomized trials and comparison studies based on PC status should be conducted, and further research on the molecular and genetic profile may help patients with Pc-only mCRC.

\section{Abbreviations}


CRC.colorectal cancer

mCRC.metastatic colorectal cancer

$P C$ : peritoneal carcinomatosis

LiM: liver metastasis

LuM: lung metastasis

OS: overall survival

PFS: progression-free survival

FOLFOX: folinic acid + fluorouracil + oxaliplatin

FOLFIRI: folinic acid + fluorouracil + irinotecan

UGT1A1: uridine diphosphate glucuronosyltransferase 1A1

IHC: immunohistochemical

CT: computed tomography

CRS: cytoreductive surgery

HIPEC: hyperthermic intraperitoneal chemotherapy

UGT: uridine diphosphate glucuronosyltransferase

AEs: adverse events

ORR: objective response rate

ESMO: European Society for Medical Oncology

\section{Declarations}

\section{Ethics approval and consent to participate}

All aspects of this study were approved by the institutional review board of Kaohsiung Medical University Hospital. Finally, this study was a retrospective review of 206 patients with $\mathrm{mCRC}$, and written informed consent was obtained from all patients (KMUHIRB-E(I)-20200036).

\section{Consent for publication}

Not applicable.

\section{Availability of data and material}

Not applicable.

\section{Competing interests}

The authors declare no conflict of interest.

\section{Acknowledgments}

None.

\section{Authors' contributions}


$\mathrm{C}-\mathrm{CL}$, being the first author of this manuscript, designed this study, analyzed the data, and wrote the manuscript. T-KC, Y-CC, H-LT, C-WH, W-CS, C-JM, T-CY, and P-JC made substantial contributions in terms of the data acquisition, interpretation and statistical analyses, in addition to assisting with the manuscript preparation. J-YW, being the corresponding author for this manuscript, also participated in the study design and coordination, in addition to making critical revisions to the manuscript. All authors have reviewed and approved submission of the final version of the manuscript.

\section{Funding}

This work was supported by grants from the Ministry of Science and Technology (MOST 109-2314-B-037035, MOST 109-2314-B-037-040, MOST 109-2314-B-037-046-MY3) and the Ministry of Health and Welfare (MOHW107-TDU-B-212-123006, MOHW107-TDU-B-212-114026B, MOHW108-TDU-B-212-133006, MOHW109-TDU-B-212-134026, MOHW109-TDU-B-212-114006) and funded by the health and welfare surcharge on tobacco products. It was also funded by Kaohsiung Medical University (KMU) Hospital (KMUH107-7M22, KMUH109-9R32, KMUH109-9R33, KMUH109-9R34, KMUH109-9M30, KMUH109-9M31, KMUH109-9M32, KMUH109-9M33, KMUHS10903, KMUHSA10903, KMUH-DK(C)110010, KMUHDK(B)110004-3), the KMU Center for Cancer Research (KMU-TC109A04-1). and a KMU Center for Liquid Biopsy and Cohort Research Center grant (KMU-TC109B05). Additionally, this study was supported by a grant from the Taiwan Precision Medicine Initiative, Academia Sinica, Taiwan.

\section{References}

1. WHO, cancer today, https://gco.iarc.fr/today/home; (Accessed on Feb 1, 2021)

2. Torre LA, Bray F, Siegel RL, Ferlay J, Lortet-Tieulent J, Jemal A. Global cancer statistics, 2012. CA Cancer J Clin. 2015 Mar;65(2):87-108. https://doi.org/10.3322/caac.21262.

3. Zarour LR, Anand S, Billingsley KG, Bisson WH, Cercek A, Clarke MF, Coussens LM, Gast CE, Geltzeiler CB, Hansen L, Kelley KA, Lopez CD, Rana SR, Ruhl R, Tsikitis VL, Vaccaro GM, Wong MH, Mayo SC. Colorectal Cancer Liver Metastasis: Evolving Paradigms and Future Directions. Cell Mol Gastroenterol Hepatol. 2017 Jan 20;3(2):163-173. https://doi.org/10.1016/j.jcmgh.2017.01.006.

4. NCCN, “Colon Cancer NCCN 2020 Ver. 4, 2020, (Accessed on Feb 1, 2021)

5. Chang TK, Tsai HL, Su WC, Huang CW, Yeh YS, Ma CJ, Wang JY. The clincopathological variables to differentiate the nature of isolated pulmonary nodules in patients who received curative surgery for colorectal cancer. Asian J Surg. 2019 Feb;42(2):425-432.

https://doi.org/10.1016/j.asjsur.2018.08.002.

6. Franko J. Therapeutic efficacy of systemic therapy for colorectal peritoneal carcinomatosis: Surgeon's perspective. Pleura Peritoneum. 2018 Mar 16;3(1):20180102. https://doi.org/10.1515/pp2018-0102.

7. Chuang SC, Huang CW, Chen YT, Ma CJ, Tsai HL, Chang TK, Su WC, Hsu WH, Kuo CH, Wang JY. Effect of KRAS and NRAS mutations on the prognosis of patients with synchronous metastatic 
colorectal cancer presenting with liver-only and lung-only metastases. Oncol Lett. 2020 Sep;20(3):2119-2130. https://doi.org/10.3892/ol.2020.11795.

8. Lu CY, Huang CW, Hu HM, Tsai HL, Huang CM, Yu FJ, Huang MY, Chang SF, Huang ML, Wang JY. Prognostic advantage of irinotecan dose escalation according to uridine diphosphate glucuronosyltransferase 1A1 (UGT1A1) genotyping in patients with metastatic colorectal cancer treated with bevacizumab combined with 5-fluorouracil/leucovorin with irinotecan in a first-line setting. Transl Res. 2014 Aug;164(2):169-76. https://doi.org/10.1016/j.trsl.2013.12.009.

9. Tsai HL, Huang CW, Lin YW, Wang JH, Wu CC, Sung YC, Chen TL, Wang HM, Tang HC, Chen JB, Ke TW, Tsai CS, Huang HY, Wang JY. Determination of the UGT1A1 polymorphism as guidance for irinotecan dose escalation in metastatic colorectal cancer treated with first-line bevacizumab and FOLFIRI (PURE FIST). Eur J Cancer. 2020 Oct;138:19-29. https://doi.org/10.1016/j.ejca.2020.05.031.

10. Hsieh YC, Chang TK, Su WC, Huang CW, Tsai HL, Chen YC, Li CC, Chen PJ, Yin TC, Ma JC, Wang JY. UGT1A1 Polymorphism for Irinotecan Dose Escalation in Patients with BRAF-Mutated Metastatic Colorectal Cancer Treated with First-Line Bevacizumab and FOLFIRI. J Oncol, https://doi.org/10.1155/2021/6686517.

11. Therasse P, Arbuck SG, Eisenhauer EA, Wanders J, Kaplan RS, Rubinstein L, Verweij J, Van Glabbeke M, van Oosterom AT, Christian MC, Gwyther SG. New guidelines to evaluate the response to treatment in solid tumors. European Organization for Research and Treatment of Cancer, National Cancer Institute of the United States, National Cancer Institute of Canada. J Natl Cancer Inst. 2000 Feb 2;92(3):205-16. https://doi.org/10.1093/jnci/92.3.205.

12. Yeh YS, Tsai HL, Huang CW, Wang JH, Lin YW, Tang HC, Sung YC, Wu CC, Lu CY, Wang JY.

Prospective analysis of UGT1A1 promoter polymorphism for irinotecan dose escalation in metastatic colorectal cancer patients treated with bevacizumab plus FOLFIRI as the first-line setting: study protocol for a randomized controlled trial. Trials. 2016 Jan 25;17:46. https://doi.org/10.1186/s13063-016-1153-3.

13. Arnold D, Stein A. New developments in the second-line treatment of metastatic colorectal cancer: potential place in therapy. Drugs. 2013 Jun;73(9):883-91. https://doi.org/10.1007/s40265-013-00765.

14. Narasimhan V, Ooi G, Michael M, Ramsay R, Lynch C, Heriot A. Colorectal peritoneal metastases: pathogenesis, diagnosis and treatment options - an evidence-based update. ANZ J Surg. 2020 Sep;90(9):1592-1597. https://doi.org/10.1111/ans.15796.

15. Jayne DG, Fook S, Loi C, Seow-Choen F. Peritoneal carcinomatosis from colorectal cancer. Br J Surg. 2002 Dec;89(12):1545-50. https://doi.org/10.1046/j.1365-2168.2002.02274.x.

16. Koppe MJ, Boerman OC, Oyen WJ, Bleichrodt RP. Peritoneal carcinomatosis of colorectal origin: incidence and current treatment strategies. Ann Surg. 2006 Feb;243(2):212-22. https://doi.org/10.1097/01.sla.0000197702.46394.16.

17. Douillard JY, Siena S, Cassidy J, Tabernero J, Burkes R, Barugel M, Humblet Y, Bodoky G, Cunningham D, Jassem J, Rivera F, Kocákova I, Ruff P, Błasińska-Morawiec M, Šmakal M, Canon JL, 
Rother M, Oliner KS, Tian Y, Xu F, Sidhu R. Final results from PRIME: randomized phase III study of panitumumab with FOLFOX4 for first-line treatment of metastatic colorectal cancer. Ann Oncol. 2014 Jul;25(7):1346-1355. https://doi.org/10.1093/annonc/mdu141.

18. Kerscher AG, Chua TC, Gasser M, Maeder U, Kunzmann V, Isbert C, Germer CT, Pelz JO. Impact of peritoneal carcinomatosis in the disease history of colorectal cancer management: a longitudinal experience of 2406 patients over two decades. Br J Cancer. 2013 Apr 16;108(7):1432-9. https://doi.org/10.1038/bjc.2013.82.

19. Schwartzberg LS, Rivera F, Karthaus M, Fasola G, Canon JL, Hecht JR, Yu H, Oliner KS, Go WY. PEAK: a randomized, multicenter phase II study of panitumumab plus modified fluorouracil, leucovorin, and oxaliplatin (mFOLFOX6) or bevacizumab plus mFOLFOX6 in patients with previously untreated, unresectable, wild-type KRAS exon 2 metastatic colorectal cancer. J Clin Oncol. 2014 Jul 20;32(21):2240-7. https://doi.org/10.1200/JC0.2013.53.2473.

20. Huang CQ, Min Y, Wang SY, Yang XJ, Liu Y, Xiong B, Yonemura Y, Li Y. Cytoreductive surgery plus hyperthermic intraperitoneal chemotherapy improves survival for peritoneal carcinomatosis from colorectal cancer: a systematic review and meta-analysis of current evidence. Oncotarget. $2017 \mathrm{Apr}$ 27;8(33):55657-55683. https://doi.org/10.18632/oncotarget.17497.

21. Quénet F, Elias D, Roca L, Goéré D, Ghouti L, Pocard M, Facy O, Arvieux C, Lorimier G, Pezet D, Marchal F, Loi V, Meeus P, Juzyna B, de Forges H, Paineau J, Glehen O; UNICANCER-GI Group and BIG Renape Group. Cytoreductive surgery plus hyperthermic intraperitoneal chemotherapy versus cytoreductive surgery alone for colorectal peritoneal metastases (PRODIGE 7): a multicentre, randomised, openlabel, phase 3 trial. Lancet Oncol. 2021 Feb;22(2):256-266. https://doi.org/10.1016/S14702045(20)30599-4.

22. Loupakis F, Moretto R, Aprile G, Muntoni M, Cremolini C, lacono D, Casagrande M, Ferrari L, Salvatore L, Schirripa M, Rossini D, De Maglio G, Fasola G, Calvetti L, Pilotto S, Carbognin L, Fontanini G, Tortora G, Falcone A, Sperduti I, Bria E. Clinico-pathological nomogram for predicting BRAF mutational status of metastatic colorectal cancer. Br J Cancer. 2016 Jan 12;114(1):30-6. https://doi.org/10.1038/bjc.2015.399.

23. Tie J, Gibbs P, Lipton L, Christie M, Jorissen RN, Burgess AW, Croxford M, Jones I, Langland R, Kosmider S, McKay D, Bollag G, Nolop K, Sieber OM, Desai J. Optimizing targeted therapeutic development: analysis of a colorectal cancer patient population with the BRAF(V600E) mutation. Int J Cancer. 2011 May 1;128(9):2075-84. https://doi.org/10.1002/ijc.25555.

24. Lu CY, Huang CW, Wu IC, Tsai HL, Ma CJ, Yeh YS, Chang SF, Huang ML, Wang JY. Clinical Implication of UGT1A1 Promoter Polymorphism for Irinotecan Dose Escalation in Metastatic Colorectal Cancer Patients Treated with Bevacizumab Combined with FOLFIRI in the First-line Setting. Transl Oncol. 2015 Dec;8(6):474-9. https://doi.org/10.1016/j.tranon.2015.11.002.

25. Ursem C, Atreya CE, Van Loon K. Emerging treatment options for BRAF-mutant colorectal cancer. Gastrointest Cancer. 2018;8:13-23. https://doi.org/10.2147/GICTT.S125940. 
26. Yoon HH, Shi Q, Alberts SR, Goldberg RM, Thibodeau SN, Sargent DJ, Sinicrope FA; Alliance for Clinical Trials in Oncology. Racial Differences in BRAF/KRAS Mutation Rates and Survival in Stage III Colon Cancer Patients. J Natl Cancer Inst. 2015 Jul 9;107(10):djv186. https://doi.org/10.1093/jnci/djv186.

27. Nordlinger B, Sorbye H, Glimelius B, Poston GJ, Schlag PM, Rougier P, Bechstein WO, Primrose JN, Walpole ET, Finch-Jones M, Jaeck D, Mirza D, Parks RW, Collette L, Praet M, Bethe U, Van Cutsem E, Scheithauer W, Gruenberger T; EORTC Gastro-Intestinal Tract Cancer Group; Cancer Research UK; Arbeitsgruppe Lebermetastasen und-tumoren in der Chirurgischen Arbeitsgemeinschaft Onkologie (ALM-CAO); Australasian Gastro-Intestinal Trials Group (AGITG); Fédération Francophone de Cancérologie Digestive (FFCD). Perioperative chemotherapy with FOLFOX4 and surgery versus surgery alone for resectable liver metastases from colorectal cancer (EORTC Intergroup trial 40983): a randomised controlled trial. Lancet. 2008 Mar 22;371(9617):1007-16. https://doi.org/10.1016/S0140-6736(08)60455-9.

28. Pawlik TM, Scoggins CR, Zorzi D, Abdalla EK, Andres A, Eng C, Curley SA, Loyer EM, Muratore A, Mentha G, Capussotti L, Vauthey JN. Effect of surgical margin status on survival and site of recurrence after hepatic resection for colorectal metastases. Ann Surg. 2005 May;241(5):715-22, discussion 722-4. https://doi.org/10.1097/01.sla.0000160703.75808.7d.

29. Yedibela S, Klein P, Feuchter K, Hoffmann M, Meyer T, Papadopoulos T, Göhl J, Hohenberger W. Surgical management of pulmonary metastases from colorectal cancer in 153 patients. Ann Surg Oncol. 2006 Nov;13(11):1538-44. https://doi.org/10.1245/s10434-006-9100-2.

30. Okumura S, Kondo H, Tsuboi M, Nakayama H, Asamura H, Tsuchiya R, Naruke T. Pulmonary resection for metastatic colorectal cancer: experiences with 159 patients. J Thorac Cardiovasc Surg. 1996 Oct;112(4):867-74. https://doi.org/10.1016/S0022-5223(96)70085-5.

31. Franko J, Ibrahim Z, Gusani NJ, Holtzman MP, Bartlett DL, Zeh HJ 3rd. Cytoreductive surgery and hyperthermic intraperitoneal chemoperfusion versus systemic chemotherapy alone for colorectal peritoneal carcinomatosis. Cancer. 2010 Aug 15;116(16):3756-62.

https://doi.org/10.1002/cncr.25116.

32. Franko J, Shi Q, Goldman CD, Pockaj BA, Nelson GD, Goldberg RM, Pitot HC, Grothey A, Alberts SR, Sargent DJ. Treatment of colorectal peritoneal carcinomatosis with systemic chemotherapy: a pooled analysis of north central cancer treatment group phase III trials N9741 and N9841. J Clin Oncol. 2012 Jan 20;30(3):263-7. https://doi.org/10.1200/JC0.2011.37.1039.

\section{Tables}

Due to technical limitations, table 1 and 2 is only available as a download in the Supplemental Files section.

\section{Figures}


2014.1.1-2018.12.31

Stage IV mCRC patients (data

from cancer center) $(n=398)$

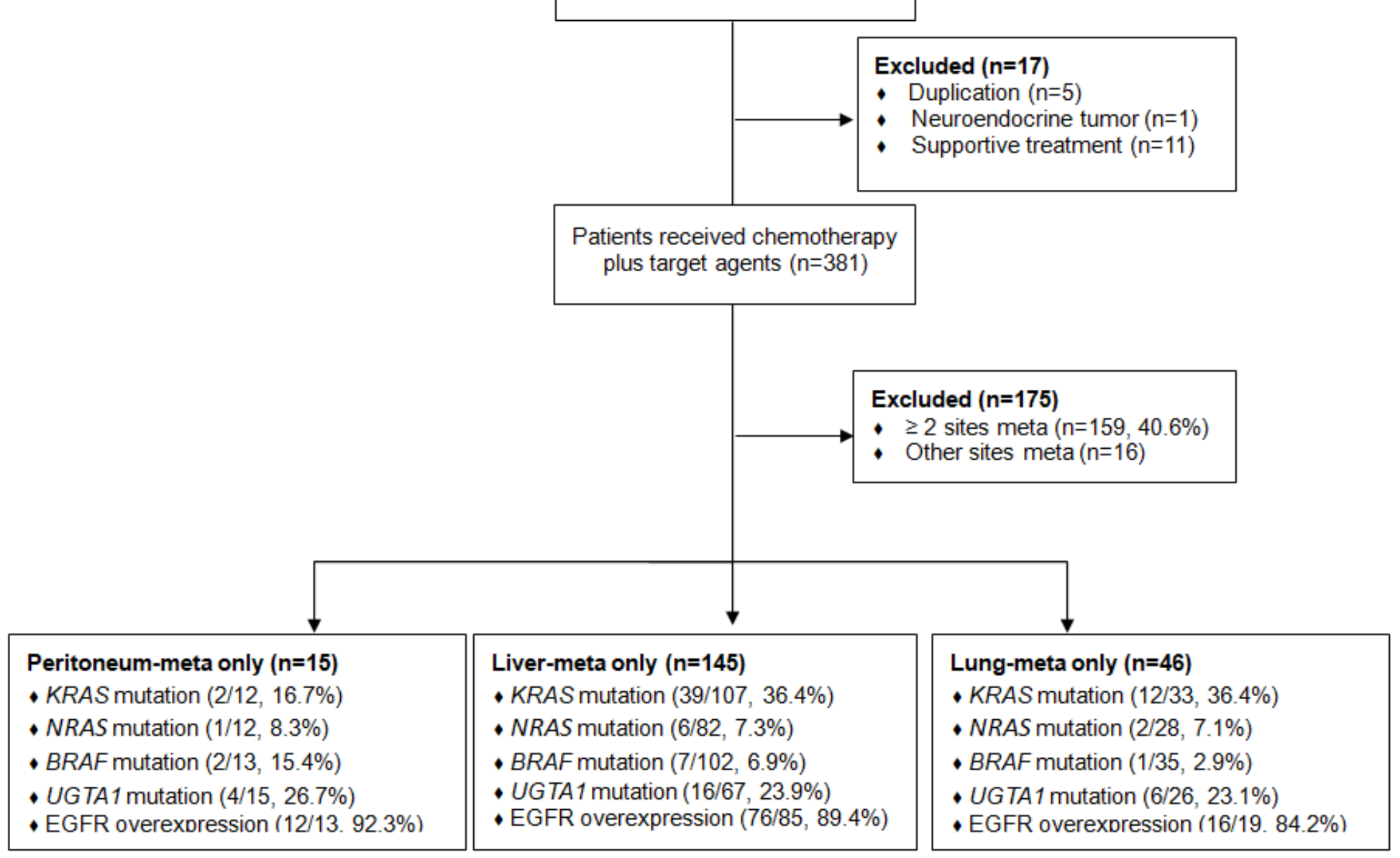

\section{Figure 1}

CONSORT flow chart for the 398 patients with mCRC; data were collected from Kaohsiung Medical University Hospital cancer center 2014.1.1-2018.12.31). 


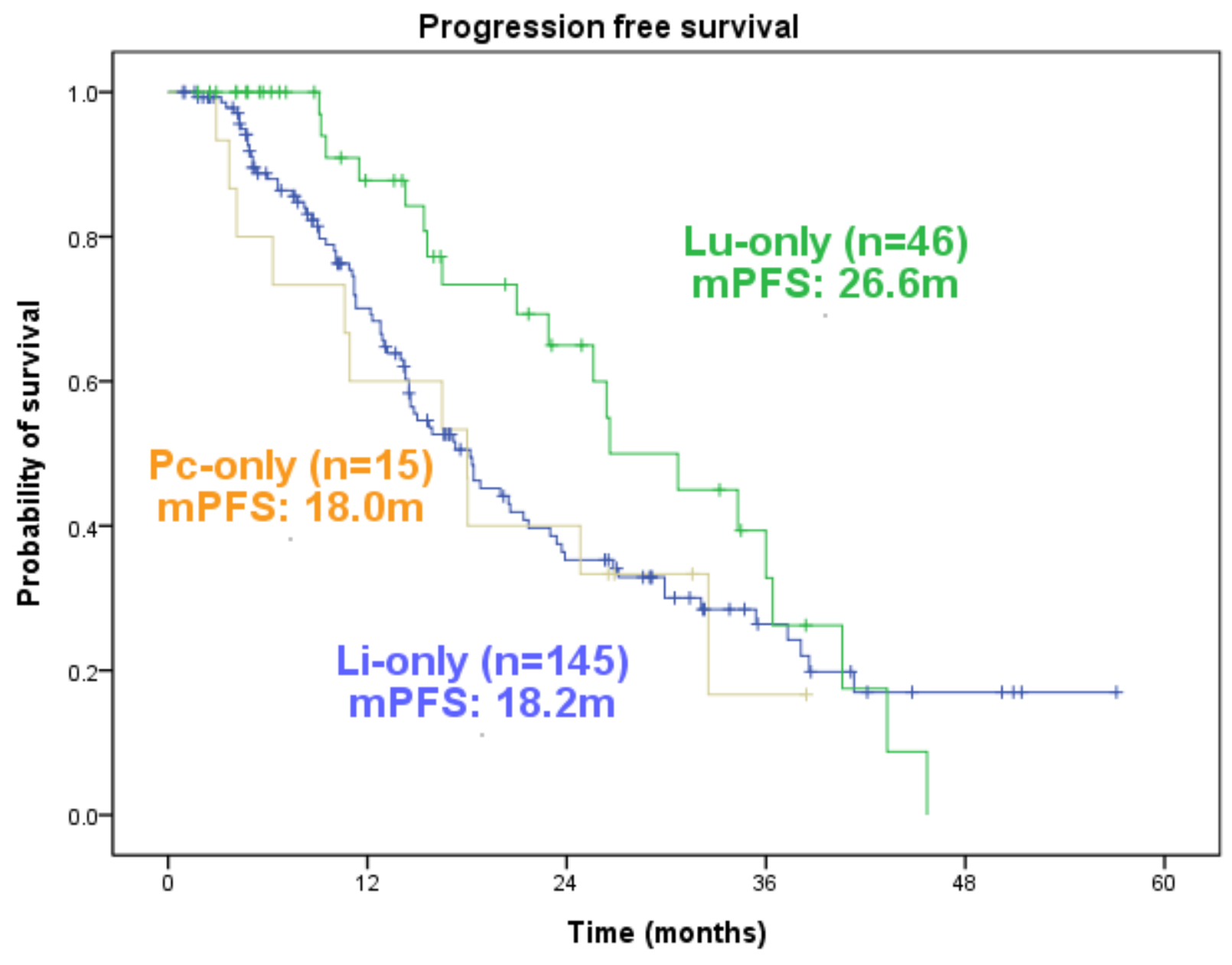

Figure 2

Kaplan-Meier analysis of progression-free survival in all 206 patients with mCRC. PC-only versus LiMonly versus Lu-only mCRC $=18.0$ versus 18.2 versus 26.6 months $(P=0.194)$. mPFS, median progression-free survival 


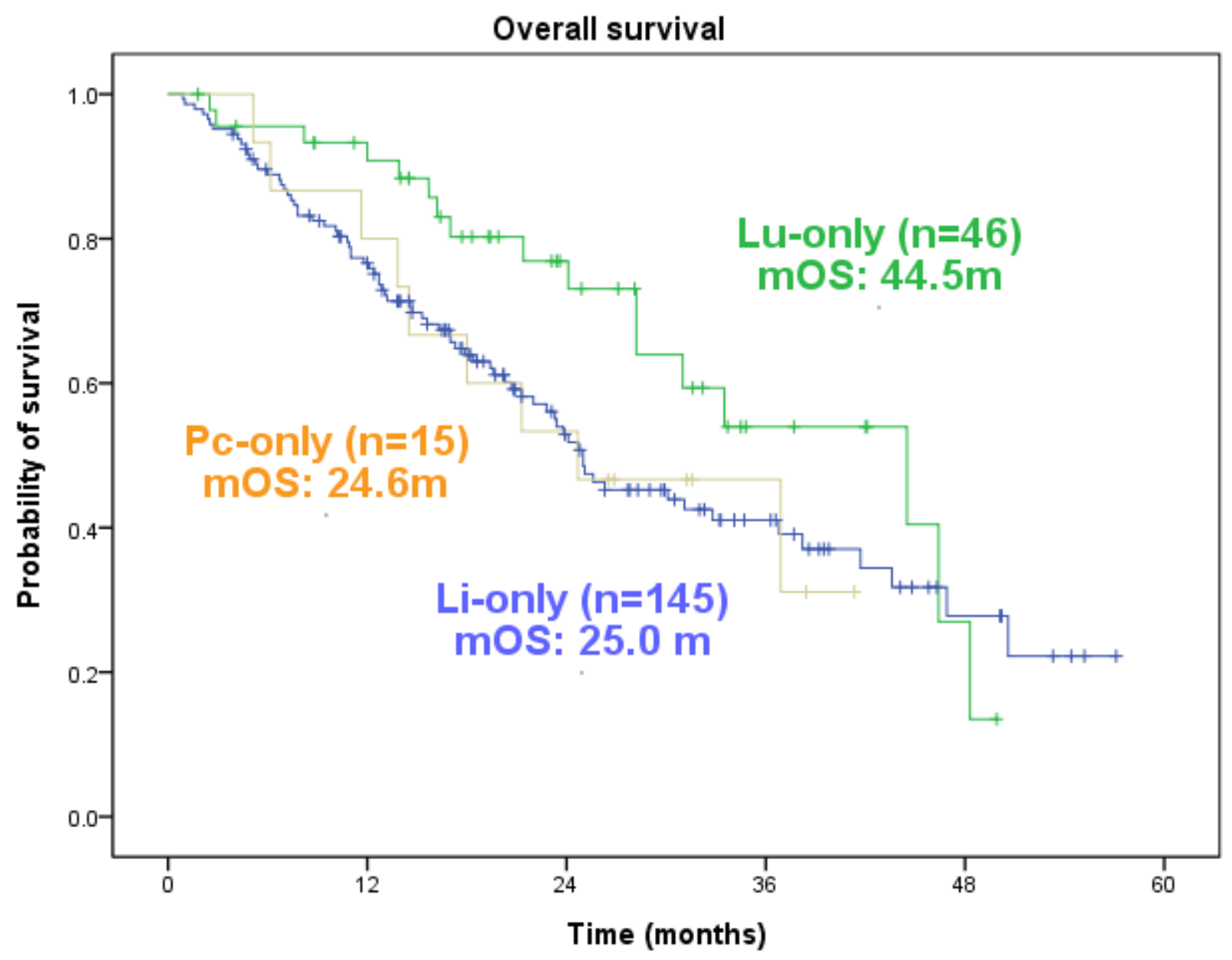

Figure 3

Kaplan-Meier analysis of overall survival in all 206 patients with mCRC. PC-only versus LiM-only versus LuM-only $\mathrm{mCRC}=24.6$ versus 25.0 versus 44.5 months $(P=0.191) . \mathrm{mOS}$, median overall survival

\section{Supplementary Files}

This is a list of supplementary files associated with this preprint. Click to download.

- Table1.tif

- Table2.tif 